\title{
LA EDUCACIÓN QUE MERECEMOS NO ES LA EDUCACIÓN QUE TENEMOS: EL PROBLEMA DE LA FALTA DE CALIDAD EN LA EDUCACIÓN BÁSICA Y SECUNDARIA EN COLOMBIA 1903-1933
}

\author{
THE EDUCATION WE DESERVE IS NOT THE EDUCATION WE HAVE: THE \\ PROBLEM OF LACK OF QUALITY IN PRIMARY AND SECONDARY EDUCATION \\ IN COLOMBIA 1903-1933
}

\author{
Rodrigo Hernán Torrejano-Vargas ${ }^{1}$
}

\section{RESUMEN}

El propósito de este artículo es identificar y explicar las causas de la baja calidad de la educación primaria y secundaria en Colombia durante los treinta años de dominio del partido conservador a principios del siglo XX, detectadas por varios intelectuales de la época, quienes argumentaron que el problema procedía de la mezcla de factores de índole eugenésico propios de la mezcla racial y cultural resultante de la época de la colonia, con la inexistencia de una facultad de estudios superiores que formara docentes altamente calificados, la falta de reconocimiento social del docente y la pobre remuneración de los mismos, motivo por el cual propusieron la realización de una reforma estructural del sistema educativo que se apalancara en la creación de la carrera universitaria de profesor y en la importación de los preceptos pedagógicos de la Escuela Nueva.

Palabras clave: Degeneración racial; desnutrición; vocación profesional; escuela nueva; ciencias de la educación; calidad de la educación.

ABSTRACT

This article identifies and explains the grounds of low quality in primary and secondary education during the thirty-year of conservative party government in the early 20th century in Colombia. Several intellectuals from that period identified these grounds and argued that such problems stemmed from the mix of eugenic factors related to racial and cultural amalgam that originated during the colonial period, the lack of high education faculties for teachers, the lack of social recognition of teachers and their low salaries. Those intellectuals proposed a structural reform to the educational system that could rely on the creation of a University degree for teachers and the implementation of pedagogic ideas from the New School.

Keywords: Racial degeneration; malnutrition; vocation; New School; education sciences; quality of education.

Tipología: Artículo de Investigación Científica y Tecnológica

Fecha de recepción: 29/04/2015

Fecha de aceptación: 13/07/2015

Como citar este artículo: Torrejano, R. (2015). La educación que merecemos no es la educación que tenemos: el problema de la falta de calidad en la educación básica y secundaria en Colombia 1903-1933. Jangwa Pana, 14, 59 - 74.

1. Licenciado en Ciencias Sociales, Universidad Francisco José de Caldas. Magister en Historia, Universidad Externado de Colombia. Docente investigador Universidad Jorge Tadeo Lozano, miembro del Grupo de Investigación Mente, Lenguaje y Sociedad. Docente investigador Corporación Universitaria Republicana, Líder del Grupo de Investigación Derecho, Sociedad y Desarrollo. Colombia. Correo: rtorrejano@gmail.com; rodrigo. torrejano@utadeo.edu.co 
El nuevo sistema sacude la inteligencia, despierta, la lleva a la acción, pone en movimiento todas sus facultades; no pretende hacer niños sabios, sino niños capaces, comprensivos. El niño podrá olvidar lo que se ha aprendido-lo olvidará menos que cuando sólo lo memorizó sin comprenderlo-, pero en si quedará la disciplina mental, la capacidad desarrollada, la facultad de encontrar lo que olvidó

Agustín Nieto Caballero

\section{INTRODUCCIÓN}

- Por qué se tiene una educación de poca cali$\zeta$ dad? A la hora de tratar de esclarecer las causas de la cuestionable calidad de la educación en Colombia varios intelectuales asumieron el reto de encontrarle respuesta a esta encrucijada con la preclara intención de diseñar y formular una salida institucional de corto y largo plazo, de lo contrario seguía corriéndose el riesgo de perder cada vez más distancia frente a los avances y logros de las naciones más avanzadas del mundo capitalista. La respuesta de personas como Miguel Jiménez, Luis López de Mesa, Gabriel Anzola Gómez, Agustín Nieto Caballero, Tomás Cadavid, Samuel Araujo y Tomás Rueda entre otros, fueron contundentes en llamar la atención sobre factores que, si bien distaban de ser un descubrimiento absoluto, permanecían y estaban convirtiéndose en un "incurable" dolor de cabeza.

Para este selecto grupo de colombianos las causas de la cuestionable calidad de la educación estaban perfectamente establecidas: mezcla racial, ausencia de vocación profesional, inexistencia de reconocimiento social de la profesión docente, deterioro de la formación normalista, bajos salarios, carencia de recursos para dotar adecuadamente las escuelas y el desempeño de la actividad docente por parte de los estudiantes académicamente menos favorecidos del sistema educativo. Con algo de más detalle sus planteamientos al respecto serán el tema del presente trabajo. Todo esto con el ánimo de contribuir a la identificación y el análisis de algunas de las causas que todavía pesan en la existencia de una educación básica y secundaria de poca calidad.

\section{La calidad es cuestión de raza}

Uno de los intelectuales de la época que se interesó por el estado y el porvenir de la educación nacional fue el médico psiquiatra boyacense Miguel Jiménez López (1875-1955), enmarcado en el discurso cientificista apoyado en los avances de la medicina y la biología, sobre manera, en el contexto de la eugenesia (Charry, 2011), fue contradictor de la practica pedagógica anacrónica con la que aún se trabajaba en las instituciones educativas públicas y privadas, totalmente de espaldas a los requerimientos de una sociedad dinámica que urgía individuos alertas y ágiles para resolver los retos de la vida personal, productiva y ética de manera expedita.

A este respecto fue contundente con los planteamientos que pronunció frente a la Asamblea de estudiantes reunida en el Teatro Municipal de Bogotá el 21 de mayo de 1920 en los que anotó:

Que nuestra educación es deficiente en todas sus etapas y en todos sus ramos; que las más de las instituciones educativas y docentes de nuestra patria no llenan la misión de formar hombres completos para la lucha por la vida, es una vieja verdad que todos los días se denuncia y que nunca se remedia [...] no sabéis, los más de vosotros, jóvenes estudiantes, a donde vais con vuestros veinte años, porque en este albor de la existencia dejasteis de aprender dos cosas esenciales: pensar y obrar [...] muchos libros han pasado por vuestra cabeza, pero han quedado sin cultivo los dos grandes poderes del hombre: "la inteligencia y la voluntad". (Jiménez, 1920a, p. 70) 
Con un poco más de detalle, el anacronismo de la educación colombiana era la enfermedad que venía padeciendo el sistema desde mucho tiempo atrás, con la existencia de un síntoma muy visible: jóvenes incapaces de sortear la vida, resultado inexorable de la contaminada práctica de dejar a un lado la capacitación de la mente. Enseñar a pensar, que era el prerequisito para saber obrar, era precisamente lo que nunca se había adelantado. La inteligencia se había dejado de cortejar porque la enseñanza nunca había apuntado hacia su utilización vivencial o práctica, por el contrario, esta había quedado reducida y restringida a la enseñanza libresca, esa que se interesa únicamente por la adquisición y almacenamiento mental de contenidos.

Dicha enfermedad recaía, en buena proporción, sobre los hombros del sistema imperante de formación de profesores. La escuela normal estaba lejos de preparar y ofrecer profesionales idóneos, acordes con la modernización capitalista en vista de su anotada condición libresca, por eso era imperioso un tratamiento radical consistente en el intercambio cultural con los países avanzados del mundo, en el que un equipo seleccionado de personas viajaran a aprender en el exterior y un equipo docente de extranjeros de reconocida calidad que arribaran a Colombia con el ánimo de impartir sus conocimientos y experiencias.

La confianza en el patrimonio académico externo, en la tradición científica pedagógica mundial, sin que nunca mencionara algún país en especial, sino solo unas condiciones de simetría cultural bastante amplias y vagas como idioma y mentalidad, fue, simultáneamente, una señal de desconfianza frente al trabajo pedagógico doméstico. Para Jiménez López y otros intelectuales, bastante más relacionados con la educación, como Agustín Nieto Caballero, Gabriel Anzola Gómez y Simón Araujo, el remedio para la educación de baja calidad era la apertura intelectual, el flujo libre de ideas e intelectuales de uno a otro lado del océano, en lo que podría denominarse un proceso de integración cultural con los mercados más desarrollados.
Por supuesto, no sobra mencionar que junto con la preocupación por el sistema de formación de los profesores colombianos, este médico boyacense, de filiación conservadora, estimó la presencia de otra serie de elementos que coadyuvaban al decaimiento de la calidad de la educación, esta vez más de la mano con las condiciones emocionales, ambientales y biológicas de los estudiantes, a saber: disminución del interés por el estudio y el debilitamiento físico de los estudiantes, por condiciones de vida malsanas y enfermedades.

La reflexión anterior hizo parte de un conjunto de disquisiciones más generales enfocadas hacia el espinoso tema de las cualidades físicas y culturales de la población colombiana frente a las características de ciertas poblaciones nativas del hemisferio norte avanzado, tanto en Europa como América, puesto que para Jiménez López en Colombia y en la América equinoccial resultaba revelador el proceso de "depresión del producto humano".

La degeneración física de la población colombiana estaba expresada en la inferioridad anatómica de talla, peso y cerebro, al indicar los estudios adelantados en este último acápite que el índice cefálico de un colombiano oscilaba entre 82 y 85 y esto lo acercaba a lo que la craneometría considera característica degenerativa (Jiménez, 1920b).

En lo que respecta a la degeneración intelectual, acotó que el signo patológico más dramático del fenómeno era la cantidad creciente de enfermedades mentales al tomar cifras levantadas por el doctor Maximiliano Rueda, médico del "asilo de locos": "en el primer lustro del siglo actual el promedio de locos varones fue de 82 por año; en el segundo lustro nunca ha sido menor de 150, y en el tercero pasa de 200 anuales" (Jiménez, 1920b, p. 22).

Ante este sombrío y desolador panorama de la naturaleza fisiológica y cultural de la gente en Colombia, detonante de la precariedad del siste- 
ma educativo y la mediocridad de los actores del proceso de enseñanza, propuso la fórmula salvadora de la "infusión de sangre fresca", que no era una fórmula distinta a proponer la puesta en marcha de una política pública de incentivo a la inmigración externa (Jiménez, 1920a).

Al lado de la conveniencia estructural de la inmigración europea blanca para depurar el obsoleto sistema educativo nacional, también puso de su parte para la aprobación y aplicación de un conjunto de medidas oficiales tendientes a mejorar la salud y la salubridad a través de una política pública de higiene que fomentara la adquisición de una cultura del aseo personal, la alimentación nutritiva y la erradicación de prácticas sedentarias. Sin que esto fuera suficiente, le adicionó a su paquete de reformas la necesidad de una política social laboral moderna volcada sobre la tarea de satisfacer algunas demandas de la clase trabajadora, la cual, por cierto, experimentaba un ambiente de agitación y organización que iba de la mano con la introducción de la economía de enclave en el sector agrícola y minero, y la modernización de los medios de transporte (Vega, 2002; Tirado, 1985; Torrejano, 2009).

Claro está que en el planteamiento de este conjunto de soluciones para el mejoramiento de la calidad de la educación se encontraba acompañado de renombrados personajes de la vida política nacional de la época como Luis López de Mesa, Ministro de Educación durante una parte del mandato liberal de Alfonso López Pumarejo, quien se empeñó por una acción gubernamental integral que le brindara a la infancia inmejorables condiciones para asistir a la escuela, según se desprende de esta apreciación:

Prohibió que se les diera trabajo a los niños menores de 14 años y proclamó la educación como prioridad para los más pobres. Igualmente pensaba que sin higiene y sin alimentación adecuada seria inútil reformar la educación. De esta manera conformó co- misiones nacionales compuesta cada una por un médico, un dentista y un inspector escolar, para que visitaran las escuelas existentes en Colombia (Álvarez, 2005, p. 13).

\section{La calidad es cuestión de sueldo}

Por supuesto, las respuestas de los interesados en el asunto de la baja calidad de la educación colombiana no pararon aquí, para pocos era desconocido que también había que sopesar el factor económico de la retribución de los docentes. La literatura privada y los informes públicos dan testimonio de la baja remuneración. En 1920, el Director General de Instrucción Pública del departamento del Atlántico, Julio Labarrera, en el informe enviado al Ministerio de Instrucción, fue enfático en señalar la relación directamente proporcional que existía entre la mediocridad docente y los salarios paupérrimos que recibían, en condiciones de excesivo trabajo dado el alto número de estudiantes por docente:

No se necesita, sin embargo, mucho esfuerzo para ver que de conformidad con el dato ya anotado, cada escuela, si se exceptúan dos, está a cargo de un solo maestro, quien tendrá naturalmente que clasificar los alumnos por secciones o grupos, para poder enseñar, y que en tales condiciones es imposible una labor eficaz aunque el maestro sea un genio pedagógico [...] y están por añadidura mal remunerados como sucede en el Atlántico, donde los sueldos son verdaderamente deficientes, dado el encarecimiento de la vida cada día mayor, tenemos forzosamente que convenir que, por un cúmulo de circunstancias extrañas a las capacidades y aptitudes de los maestros, la eficacia de su labor [...] deja mucho que desear (Memoria del Ministerio de Instrucción Pública al Congreso, 1920, p. 33-34). 
Lo mismo aconteció en el departamento de Santander, pues el señor José Salazar, Director General de Instrucción llamó la atención de las autoridades nacionales sobre la falta de personas capacitadas para dedicarse a la enseñanza debido, fundamentalmente, a los bajos salarios. A continuación su punto de vista: “el mal está en la carencia de institutores graduados o de hombres aptos que se dediquen a las enseñanza escolar, pues ni los unos ni los otros quieren ya trabajar en este ramo, debido a los pequeñísimos sueldos" (Memoria del Ministerio de Instrucción pública, 1920, p. 95).

Luego, en un informe que rinde la inspección seccional de instrucción de la segunda zona escolar del departamento de Bolívar, comprendiendo los municipios de El Carmen, San Juan Nepomuceno, San Jacinto, Guamo, Zambrano, Córdoba, Ovejas y Ricaurte, seis años más adelante, en junio de 1926, en pleno periodo de la "danza de los millones" o "prosperidad al debe", en palabras de Alfonso López Pumarejo, el serio problema de la cuestionable calidad de la educación seguía atado al factor de le cuestionable calidad de los docentes en ejercicio, y este a su vez, conectado con la sobrada falta de interés que otros sectores sociales más preparados académicamente sienten por una actividad que no reporta los dividendos económicos necesarios, tal y como se observa a continuación:

Adjunto va un informe de carácter privado sobre la opinión que me merece el personal docente de esta zona, el que, en sentido pedagógico deja bastante que desear. Maestros y maestras hay que desconocen la pedagogía en absoluto u otras que aunque tengan algunas nociones desempeñan puestos que para ellos resultan pesados; pocos muy pocos son los de las agregaciones [...] que poseen suficiente preparación literaria y por lo tanto, mucho menos pedagogía [...] Sin embargo, ocasiones hay en las que con poco se encuentran personas que por su preparación literaria podrían prestar el servicio más eficientemente que otras que no lo poseen y sin embargo influencias especiales (como los bajos sueldos por ejemplo) impiden un cambio favorable para la escuela (Carvajal, 1926).

Además, es imposible pasar por alto las desfavorables y desmotivantes condiciones materiales de trabajo de los docentes debido a la falta de útiles, que es lo mismo a identificar la falta de recursos suficientes para financiar por completo las necesidades logísticas de la educación, según se desprende de este relato: "utilizaban las piedras como pizarras sobre las que hacían escribir a los alumnos con guijarros aguzados; daban las lecciones de escritura sobre la arena en la orilla de un río" (Helg, 2001, p. 54).

\section{La calidad es cuestión de falta de preparación y vocación docente}

Aunque, por supuesto, los reportes acerca de la cuestionable calidad de los docentes colombianos no siempre estuvieron atados al factor de la baja remuneración, adicionalmente, algunas personas, vinculadas con la cultura y el gobierno de las regiones, como Alfonso Mora en el departamento de Antioquia, fue muy preciso y contundente en señalar, en tono bastante clerical, que la ausencia de un sentido de identidad con el ejercicio de la labor docente era el factor que más incidía en su irregular desempeño; profesores sin pasión, sin dotes, carentes de vocación, o docentes "nominales" era lo que estaba ocasionando el daño irreparable del sistema educativo, y un daño colateral, la agudización del deterioro de la imagen del profesor. Este su punto de vista literal al respecto:

En resumen: en esta obra de la educación moral [...] fracasan siempre los maestros que carecen de dotes pedagógicas y vocación: y he aquí 
un punto negro en la conciencia de aquellos que ejercen la profesión sin haber sido ungidos para ella; una gravísima preocupación para aquellos maestros que son meramente nominales y que enseñan, no en calidad de apóstoles de la ciencia y de la virtud, sino porque la situación está muy difícil y no tiene más que hacer (Mora, 1918, p. 104).

La falta de vocación a la que aludía Alfonso Mora pudo corroborarse en las sucesivas visitas de los inspectores provinciales de educación en la región del nordeste antioqueño, donde encontraron profesores perezosos e incapaces que no preparaban las clases ni tenían idea clara de los contenidos del programa escolar de cada asignatura, según se desprende del siguiente informe:

Hay en la Provincia del Nordeste maestros que no preparan sus lecciones con anticipación, que no tienen formados los programas de las asignaturas y que, por esta razón, no pueden trabajar con orden, con actividad, variedad y precisión, ni emplear enseñanza racional. Un impreparado pierde el tiempo, y lo peor es que lo hace perder a todos los niños que se le confíen. También hay en Nordeste y en el resto del Departamento, maestros que duermen sobre el diploma que les expidió la Escuela Normal. Quien no avanza, retrocede [...] quizá llegue el momento de una revisión de grados que los despierte de ese fatal letargo [...] (Informe de los inspectores generales, 1918, p. 220).

Resulta, entonces, que para los inspectores del departamento de Antioquia un número indeterminado de docentes no quería lo que hacía y no tenía idea de lo que hacía, por lo que era imposible esperar resultados académicos halagadores, pero así mismo pasaba que algunos docentes comprometidos, enamorados de su profesión, pecaban en contra de la calidad cuando dejaban en claro que preferían la educación memorística en lugar de aquella que invitaba y enseñaba a pensar. Con suma frecuencia y no solamente desde estos primeros años del siglo XX, sino desde los años mozos de la república, sectores sociales involucrados con la cultura y las autoridades nacionales venían empeñados en diezmar la costumbre de regirse por el método memorístico, no obstante, la costumbre estaba fuertemente arraigada en la práctica laboral cotidiana. Así lo revela el informe de visita de inspección que practicó José Villegas en la provincia de Buga, departamento del Valle del Cauca durante el primer trimestre de 1915, en el que dejó plasmado este diagnóstico:

La enseñanza que se da en los establecimientos oficiales no es aun plenamente satisfactoria, ni en la parte instructiva ni en la educativa. No se pueden arrancar del todo las tendencias a los anticuados sistemas, y ese aferramiento a la letra de los libros de texto, que tanto se opone a la iniciativa y al esfuerzo mental propio de los alumnos que es la gimnasia del desarrollo de las más altas facultades del espíritu. He aquí un ejemplo; si se le pide a un alumno del 4 año alguna noticia de Nariño, por ejemplo, no da respuesta alguna hasta que el maestro le da la clave, soplándole las primeras palabras del párrafo [...] el niño se suelta en seguida recitando de memoria hasta llegar a algún mal paso del que lo saca de nuevo el maestro, mediante palabras consagradas del libro, que tiene descaradamente en sus manos. Y esto pasa en aritmética, en castellano, en geografía, y en todas las materias que son objeto de la enseñanza $[. .$.$] es muy común que$ si el niño no recita textualmente de memoria, no puede dar razón de nada (Villegas, 1915, p. 499). 
Lo anterior sencillamente corrobora el informe que nueve años atrás diligenció Miguel González, inspector provincial del suroeste antioqueño, donde encontró que en el municipio de Salgar la directora de una escuela seguía empecinada en el fortalecimiento exagerado de la memoria, no sin destacar que hacia una noble labor en asuntos éticos, pues las niñas dieron muestra de buenos modales y aseo: "las niñas examinadas exhibieron poco aprovechamiento, pero mostraron compostura, atención y aseo. En los sistemas de enseñanza adoptados por la Directora, predomina el empirismo, el estudio en voz alta y el esfuerzo exagerado de la memoria [...]" (González, 1906, p. 362). Descripción que se repite con el director de la escuela urbana publica de varones del Municipio de Támesis, a quien el inspector provincial anotado le reconoce excelsas cualidades éticas pero insuficiente preparación pedagógica, lo que venía ocasionando el poco aprovechamiento de los niños (González, 1906, p. 367).

Si para "sorpresa" del científico norteamericano Isaac Holton, de visita en Colombia durante la década de 1820, los estudiantes del prestigioso Colegio del Rosario estudiaban "paseándose de un extremo a otro de los corredores, recitando en voz alta las lecciones que debían presentar" (Holton, 1981, s.p.), ya se podrán imaginar el profundo desencanto que desencadenaría la vivencia de la misma práctica en buena parte de las instituciones de educación básica y secundaria del país un siglo después, tanto que condujo al inspector provincial de Antioquia Elías Gómez después de la visita realizada al municipio de Fredonia, a sugerir que los docentes mediocres partidarios de los mecanismos memorísticos fueran separados inmediatamente de sus cargos como se pormenoriza en el siguiente informe:

De los 28 maestros visitados en este mes, 20 lo hicieron muy bien en las clases que dictaron, 2 solo presentaron trabajos aceptables y no satisfizo la práctica de 6 . Con estos 8 se ne- cesita emplear más visitas, y visitas más detenidas hasta ver si aprenden, para, en caso negativo, reemplazarlos sin consideración alguna. "Maestros que después de varios años de práctica continúan ignorando como se emplea la inducción en la enseñanza y que se aferran a los procedimientos memoriales o mecánicos, no evolucionaran y es preciso desecharlos, atendiendo al bien de los escolares" (Gómez, 1918, p. 245).

Ante este maremágnum de noticias poco halagadoras del estado de la calidad de la educación, en regiones apartadas del centro del país, como en Arauca, el Director de Instrucción, en aplicación de una vieja práctica política, acudió a las congregaciones religiosas para sortear el problema de la falta de idoneidad docente. Allí llevó a las Hijas de La Caridad de San Vicente de Paúl (Memoria del Ministerio de Instrucción Pública al Congreso, 1920, p 8).

Porque algo resulta evidente, la cantidad de profesores preparados, salidos de una Normal, con título eran la excepción en los años veinte. En el territorio nacional de Arauca habían 20 profesores de primaria sin título de los 24 registrados; en San Andrés y Providencia esta misma relación fue de 13 a 15; en Tierradentro las cantidades fueron 16 de 20; en el departamento de Antioquia de 1142 a 1542; en el departamento del Atlántico de 57 a 113; en el departamento andino de Boyacá, en la educación básica, la proporción fue 473 a 600 y en el departamento del Huila 103 de 162 (tabla 1). En general, puede apreciarse que, tomando en cuenta los datos de las once (11) zonas que realizaron el censo de los profesores con y sin título, (sin considerar la información del departamento de Santander por ser resultados cuestionables), tenemos que el $74 \%$ de los docentes de primaria carecían de formación pedagógica, es decir, 3014 de un universo de 4042 (tabla 1). 
Tabla 1. Número de docentes con y sin título en primaria 1920

\begin{tabular}{|c|c|c|c|}
\hline ZONA & $\mathrm{N}^{\circ}$ Profesores & $\begin{array}{c}\mathrm{N}^{\circ} \text { profesores } \\
\text { con título }\end{array}$ & $\begin{array}{c}\mathrm{N}^{\circ} \text { profesores } \\
\text { sin título }\end{array}$ \\
\hline Arauca & 24 & 4 & 20 \\
\hline Caquetá y Putumayo & 40 & N. D. & N. D. \\
\hline Casanare & 31 & N. D. & N. D. \\
\hline $\begin{array}{l}\text { San Andrés y } \\
\text { Providencia }\end{array}$ & 31 & N. D. & N. D. \\
\hline San Martín & 25 & N. D. & N. D. \\
\hline Tierradentro & 20 & 4 & 16 \\
\hline Antioquia & 1542 & 400 & 1142 \\
\hline $\begin{array}{l}\text { Atlántico* sector } \\
\text { público }\end{array}$ & 113 & 56 & 57 \\
\hline Bolívar & 53 & N.D. & N. D. \\
\hline Boyacá & 600 & 127 & 473 \\
\hline Caldas* sector público & 744 & 126 & 618 \\
\hline Cauca & 294 & 110 & 184 \\
\hline Cundinamarca & N. D. & N. D. & N. D. \\
\hline Huila & 162 & 59 & 103 \\
\hline Magdalena & 212 & 43 & 169 \\
\hline Nariño & 397 & N. D. & N. D. \\
\hline Norte de Santander & 291 & N. D. & N. D. \\
\hline Santander & 445 & $445^{\star \star}$ & 0 \\
\hline Tolima & 331 & 99 & 232 \\
\hline Valle & 458 & N. D. & N. D. \\
\hline
\end{tabular}

Fuente: Memoria del Ministerio de Instrucción Pública 1920, pp. 8-113

**Cifra que puso en duda por el Director General de Instrucción Pública del departamento José Salazar, pues los datos fueron registrados por el Director General saliente Alejandro Noriega.

N. D. No hay datos

La pobre preparación académica de los docentes de las escuelas del país, o la inexistencia de dicha preparación, ciertamente fue una migraña que el Estado intentó remediar mediante la fundación de Normales, reformas del pensum de las mismas, contratación de misiones pedagógicas extranjeras, publicación de revistas y la organización en la segunda década del siglo XX de seminarios anuales de actualización pedagógica establecidos por el Ministerio de Instrucción Pública, ley 62 del 12 de diciembre de 1916, por la cual se fomentaban algunas corporaciones pe- dagógicas. Diario Oficial 15971 (Dic. 16 1916), junto con el esfuerzo de los inspectores provinciales del Ministerio de Instrucción por impartir conferencias cortas en los municipios a su cargo. Un ejemplo del esfuerzo personal de los funcionarios del ministerio de educación por mejorar la formación docente lo efectuó el inspector provincial del Departamento del Valle del Cauca José Villegas a fines del mes de julio de 1913 con una conferencia en torno a la pedagogía moderna, cuya base era despertar el interés del niño. Estas son sus apreciaciones al respecto: "El in- 
terés pedagógico consiste en excitar el apetito del espíritu del niño, en incitarle gusto por lo que se enseña, y en hacerle agradable el alimento moral e intelectual que se le infiere. Tal interés debe ser creado por el arte del maestro, y aquí es donde campea la originalidad de la invención" (Villegas, 1914, p. 237).

A lo que agregó la explicación de un método de enseñanza compuesto de tres componentes, a saber: "la invención, es decir, la elección del asunto; la disposición, la distribución de los detalles del asunto elegido, y por último, la elocución, es decir, la manera de exponer el punto determinado por la invención y clasificado por la disposición" (Villegas, 1914, p. 237).

Pero como todas estas indicaciones metodológicas podrían resultar abstractas e improcedentes, también se contó, en el departamento de Antioquia, con la publicación en la revista de instrucción pública, de una serie de recomendaciones prácticas que todos los profesores debían adoptar con el ánimo de mejorar la rutina escolar y su posicionamiento social. Con algún lujo de detalle estas fueron los encargos por saldar:

No lleguéis tarde a la escuela. Si no sois exactos, no podréis exigir que vuestros alumnos lo sean. Llegad siempre correctamente y aseados. Un maestro mal formado o sucio inspira desprecio a sus mismos discípulos [...] No os sentéis antes de ver que vuestros alumnos ocupen ya sus puestos, ni permanezcáis sentados el tiempo que dure la clase [...] No enseñéis sin haberos preparado [...] No hagáis nunca explicaciones largas. La atención del niño es débil y no podrá seguiros $[\ldots]$ No llaméis a los niños diciéndoles tú, vos, éste, aquel. Aprended sus nombres y habladles de usted [...] No pongáis a un alumno de celador de los demás [...] No seáis prodigo en regaños [...] No os burléis nunca de los niños. Ya sabéis que la burla es la ofensa más difícil de perdonar [...] No ocupéis a los niños en cosas ajenas a la escuela [...] (Consejos prácticos a los maestros de escuela, 1918, p. 263-265).

\section{Por la educación que la nación merece: reajuste a la formación docente}

Como complemento de los esfuerzos anteriores, también es oportuno mencionar que hubo quienes desde la academia levantaron su voz para señalar que el mejoramiento de la educación en el país pasaba por el cedazo de la depuración de las escuelas Normales, por lo que era imperioso que el gobierno nacional se diera a la tarea de organizar una facultad de estudios superiores que se encargara de la formación de los docentes de estas instituciones educativas, de los colegios de secundaria y los inspectores del ramo. La proposición de fundar una facultad de Ciencias de la Educación, que se sumara a la oferta universitaria en los campos de las ciencias humanas y las ciencias básicas quedó claramente estipulada en la proposición de Rafael Bernal Jiménez a principios de las década de 1930, quien en poco tiempo se convertiría en el primer rector de esta facultad y se desempeñó, adicionalmente como profesor de filosofía y sociología de las universidades del Rosario y Javeriana de Bogotá.

Más, como la preparación de maestros es imposible sin normales y las normales no pueden funcionar sin personal que los rija y sin profesorado competente, la conclusión definitiva es la de que se requería un instituto que preparara seriamente este personal. Ahora bien: este fue el principal fin propuesto al concebir la idea de la Facultad de Educación [...] (Bernal, 1949, p. 43-44).

Aunque vista con un poco más de detalle la proposición del profesor Rafael Bernal iba hasta el pun- 
to de indicar que la fundación de la facultad tampoco era la panacea si no estaba atada o vinculada con el axioma de que allí ingresaran y estudiaran jóvenes de mente brillante o por lo menos con capacidades intelectuales destacadas, su idea era que allí estuvieran los mejores bachilleres del país después de cumplir un serio y exhaustivo proceso de selección, descrito en estos términos: "Esta se verificará a base de una primera selección de alumnos-maestros, elegidos por concurso entre los más capacitados de los maestros graduados de los departamentos o de los jóvenes bachilleres que quisiesen seguir la carrera del magisterio superior" (Bernal, 1949, p. 46).

La selección de los aspirantes a conformar la facultad de ciencias de la educación sería el primer paso de un proceso de depuración mucho más ambicioso, el cual continuaba durante el tiempo de estudio profesional de tres años presupuestado para la obtención del título de licenciado. Cada año de estudio los estudiantes se someterían a una estricta evaluación que determinaría su permanencia o exclusión de la carrera. He aquí sus indicaciones al respecto:

Una segunda selección rigurosa se verificaría al terminar el primer año de estudios; tras una tercera y cuarta selección, que se verificaría al final del segundo y del tercer año, los alumnos que hubiesen aprobado todos los cursos recibirían el título de licenciado en Ciencias de la Educación. (Bernal, 1949, p. 46).

Este alto nivel de exigencia en la selección de los estudiantes y su permanencia en la facultad de educación se trató de una apuesta demasiado alta frente a las condiciones materiales y culturales imperantes por aquellos días. La posibilidad real de atraer a los mejores bachilleres dependía, en buena proporción, de los ingresos esperados en el momento del ejercicio profesional, situación que no se daba según lo reportan todos los inspectores de educación, dada la penuria fiscal de los entes administrativos encargados del financiamiento de la educación de acuerdo con la ley de 1903 que descentralizó el gasto del ramo. Sin dejar pasar el hecho de que la labor docente seguía siendo una actividad de poco o escaso reconocimiento social.

Sabiendo la existencia de estas condiciones estructurales, el profesor Bernal Jiménez salió en defensa de su tesis acotando que la mejor manera de superar el inconveniente había sido el establecimiento de un tiempo de formación teórica corto, tres años, frente a las demás carreras presentes en la oferta universitaria de la época. Así, entonces, resulta que la idea era que se diera una correlación positiva entre el corto tiempo de estudio y el salario esperado. Miremos de cerca esta sugerencia:

Podría hacerse el reparo de que el lapso de tres años de estudios universitarios sería escaso para la preparación de profesores especializados y esto puede ser fundado en principio; mas no hay que perder de vista que es humano que el tiempo requerido para una carrera sea proporcional a la remuneración que más tarde está reservada para el ejercicio de la respectiva profesión y, entre nosotros, el trabajo del profesorado se halla mal remunerado y no ve la posibilidad de que esta situación varíe en forma apreciable en poco tiempo [...] Por otra parte, hay otra consideración de carácter psicológico, y sería el no alarmar y retraer con una carrera universitaria demasiado larga, a los bachilleres y a los maestros que quisieren seguirla, teniendo muchos de ellos, la posibilidad de elegir otras carreras también universitarias (Bernal, 1949, p. 49).

Efectivamente, la proposición de Rafael Bernal adquirió forma con la expedición del decreto 1990 de 1933, que oficializó el nacimiento de la 
facultad de ciencias de la educación adscrita a la Universidad Nacional compuesta por siete secciones: "pedagogía, ciencias histórico-geográficas, ciencias físico-químicas, biología, filosofía y letras, matemáticas e idiomas" (Herrera, 1999, p. 220), convirtiéndose en el segundo esfuerzo por darle forma y fondo a la formación de docentes en el país, pues no puede echarse en saco roto el esfuerzo de Tomás Cadavid Restrepo, en el departamento de Antioquia por darle vida a la facultad o escuela de educación en la Universidad de Antioquia, creada mediante la Ordenanza 19 del 7 de abril de 1926 (Ríos, 2004, p.74), quien por cierto, al igual que Bernal Jiménez, compartió el interés de atraer las mejores mentes jóvenes del país hacia la educación pero dudaba de la viabilidad al sopesar la injerencia de los sueldos en el momento de que un joven decidiera cual carrera profesional le aportaba solides financiera en el futuro:

La remuneración que se paga actualmente a los maestros es reducida, y el que es dueño de inteligencia y de vigor, aunque matando en su alma hermosos ideales, se aleja de las aulas y va en pos de otras colocaciones que le brindan mejor producto. Quedan todavía en las filas educadoras apóstoles que desprecian estos halagos y se resuelven a vivir pobremente sirviendo a la juventud, pero estos casos no son tan numerosos que puedan llenar todos los vacíos, y así nos hallamos en una situación de verdadera crisis de maestros (Cadavid, 1927, p. 7).

Cuestión que pesaba menos en algunas destacadas instituciones del sector privado dedicadas a la formación de niños y jóvenes adinerados, donde los profesores contratados no pertenecían a los estratos socioeconómicos inferiores sino que eran miembros de la clase alta y podían acreditar formación universitaria, siendo algunos de ellos enviados al exterior en pos de su adelantamiento profesional con gastos sufragados, no sin grandes esfuerzos monetarios, por los propietarios de la institución. Esa fue la experiencia del
Gimnasio Moderno descrita por el rector Tomás Rueda Vargas en octubre de 1923:

Mas como a medida que ascienden y se perfeccionan todos los órdenes del colegio, deben ascender y perfeccionarse quienes son nervio de él [...] se hace preciso que continuemos realizando lo que ha sido uno de nuestros mayores anhelos: el envío de jóvenes profesores a completar estudios superiores en Europa, para que, viniendo luego a aplicar los nuevos conocimientos adquiridos, mantengan una corriente permanente que refresque el ambiente de nuestro instituto, evitando que caiga en la rutina y se pierda en un penoso estancamiento (Rueda Vargas, 1945, p. 52).

Determinación que no era para menos, al reconocerse, por cuenta de los propios fundadores, las directivas y mesías del proyecto del Gimnasio Moderno, que allí era donde la elite nacional estaba formándose, lo que los obligaba a ser mucho más rígidos y creativos en el tema de la capacitación de sus docentes, consideración que se desprende de la siguiente apreciación del rector Tomás Rueda:

Por vía de cargo se ha dicho que el Gimnasio se ocupa en la educación de las llamadas clases dirigentes. Es verdad, en esto reside precisamente su mérito, y ello constituye la suma de sus dificultades. Mientras la educación de las gentes que por cualquier razón ocupan posiciones ventajosas en la sociedad, no se atiende con todo el cuidado que merece, es inútil pretender que ellas influyan benéficamente en el progreso bien entendido del país [...] (Rueda Vargas, 1945, p. 52). 


\section{Por la educación que la nación merece: participación del sector privado}

Como si todos estos factores anotados en los párrafos anteriores no fueran suficientes en el hallazgo de las razones de la cuestionable calidad de la educación básica, cabe mencionar uno más, se trata de las difíciles condiciones materiales de vida de las familias de los niños de la clase baja urbana y rural para los que fueron abiertos los cupos en las escuelas de primaria de la nación. La pobreza impedía que muchas familias decidieran matricular a sus hijos, o en el mejor de los casos, creaba circunstancias adversas de asistencia que impedían el funcionamiento óptimo de sus condiciones naturales, pues con hambre y frio de nada servía el ofrecimiento de un servicio de educación, por lo menos eso lo dejó ver el comentario de Agustín Nieto Caballero a finales de 1915 con respecto a la necesidad de fundar órganos privados que subsidiaran parte representativa de los gastos de los niños pobres de las escuelas de Bogotá:

Salta a la vista que el problema de la instrucción pública no puede solucionarse con solo lucubraciones pedagógicas. O se estudia con el corazón también, o no será posible comprenderlo nunca. Mientras haya hambre y frio en la escuela, de nada servirán lo sistemas, por buenos que ellos sean [...] El primer deber de la escuela pública podría formularse así: poner a los niños en capacidad de aprender. Resuelto este problema, podemos hablar de pedagogía y de otros asuntos sociales; antes no (Nieto Caballero, 1915, p. 33-34).

Para Nieto Caballero y otros personajes de la vida económica, política y cultural, no solo de la ciudad capital, sino de todo el país, entre los que estaban los hermanos Samper Brush, los hermanos Caballero Calderón, Eduardo Santos, Luis
Cano, Nemesio Camacho, Foción Soto, Esteban Jaramillo y Santiago Ospina por dar nombres destacados, el ofrecimiento de cupos a los pobres por parte del Estado era una política pública acertada pero insuficiente. La intervención social de Estado debía acompañarse y complementarse con la injerencia del sector privado. Para tal efecto concibieron la creación de las Cajas Escolares, instituciones de beneficencia de origen decimonónico francés.

Las Cajas Escolares fueron una figura de corte asistencialista erigida en 1849 por los soldados de la Guardia Nacional francesa, quienes: “[...] sabedores de que había en París muchas familias imposibilitadas para mandar a sus hijos a los centros de instrucción por falta absoluta de ropas y calzado, resolvieron ayudar con parte de sus ahorros a estos desgraciados" (Nieto Caballero, 1915, p. 8).

La aplicación de la figura o el ejemplo asistencialista francés en Bogotá por cuenta y riesgo de la buena voluntad y los recursos humanos y monetarios de la clase alta buscaban cimentar y aclimatar entre los pobres el ambiente ideal y propicio para el adelanto y perfeccionamiento de la educación básica, lo que resultaba igual a decir que la gente rica de la ciudad, fruto de la simbiosis de expectativas ideológicas y culturales laicas y clericales, se empeñó en fortalecer la incubación de una mentalidad y actitud proclive al recibimiento y la valoración de la educación.

Con algún grado más de detalle, esta simbiosis cultural representó la fusión entre principios civiles liberales y democráticos con valores cristianos en el seno de la clase alta, en beneficio del entendido de formular un discurso y una práctica social que borrara de la mente de las personas de escasos recursos la idea de que asistir era una carga y pérdida de tiempo, y pudiera construir, por el contrario, la imagen de un activo familiar incalculable, pero eso sí, medible, y por tanto, asible en el término de la distancia, puesto que la asis- 
tencia a las clases traería consigo alimentación y vestido gratuito, que sería suministrado a través de donaciones en dinero o en especie y con la organización de un Club de Costura entre las esposas de los hombres ricos, que a la postre conformaron la Junta Directiva de las Cajas escolares, quienes dieron el siguiente parte de victoria de su novel gestión a finales de 1915: "organizaron las señoras que componen la Junta Directiva de las Cajas Escolares, un Club de Costura y [...] han logrado instalar los desayunos en diez escuelas de la ciudad. Los primeros doscientos vestidos se están repartiendo actualmente" (Nieto Caballero, 1915 , p. 31), confirmando con esta gestión, a la vez, el objeto esencial consagrado en los estatutos de fundación de la Caja, a saber: "Las Cajas Escolares tienen por objeto auxiliar en cuanto sea posible a los alumnos pobres [...] Este auxilio consistirá principalmente en alimentos, vestidos, útiles y libros adecuados al aprendizaje (Nieto Caballero, 1915, p. 38).

\section{Por la educación que la nación merece: la transformación pedagógica}

El interés de mejorar la formación académica de los docentes encontró su centro de gravedad teórico y pedagógico con los preceptos de la Escuela Nueva, por cuanto sus impulsores no dudaron en ningún momento que sus características hacían de ella la fórmula idónea para que la niñez y la juventud se formaran con los aperos formales y prácticos que una sociedad en transformación o modernización demandaba.

Dos de las personas más decididas en la adopción de la escuela Nueva como fórmula de salvación de la educación colombiana fueron por un lado, Agustín Nieto Caballero, y por otro, Gabriel Anzola Gómez, quien en 1930 escribió que la brecha entre Colombia y los países más desarrollados del mundo occidental estaba ensanchándose debido a que ésta no se había convertido en motor de desarrollo, dado que los estudiantes ca- recían de las herramientas para superar los retos de una sociedad compleja y cambiante.

Para estos dos intelectuales la educación colombiana estaba desfasada, faltaba que los niños y jóvenes fueran formados en un ambiente fecundo, donde se tuviera la oportunidad de sembrar los valores representativos de un sujeto aplicado y propositivo, por mencionar: empuje, creatividad, adaptabilidad, ingenio, agudeza y valor.

El cultivo de las cualidades del hombre propositivo permitiría el contacto real entre los conocimientos básicos y especializados con la compleja realidad nacional, puesto que se daban pasos en firme en favor de la formación de individuos capacitados para resolver los obstáculos que impedían o entorpecían transitar el camino del desarrollo económico, político y cultural. Anzola Gómez expresó esta inquietud así:

La institución primaria proporciona al niño no sólo el mínimo de conocimientos para la vida, sino las aptitudes y disposiciones para interpretarlos y servirse de ellos. Se trata no tanto de darle ciencia sino de prepararlo para recibirla, de capacitarlo para que se sirva de sí mismo como instrumento capaz de captar las múltiples manifestaciones de la vida. De ampliar su horizonte para que él mismo se adapte a las nuevas situaciones que a diario se le van presentando. Momento a momento se plantean para él, problemas nuevos de importancia vital, delante de los cuales debe reaccionar solo, con el máximo de utilidad para sí y para los demás. En esto consiste precisamente la orientación de su inteligencia, ese es su papel, resolver los problemas que a cada instante se le presentan (Anzola-Gómez, 1930, p. 148). 
Con algo de detalle, Anzola Gómez y Nieto Caballero fueron seducidos por la Escuela Nueva toda vez que estaba claramente orientada hacia la formación de individuos con mentes despiertas y curiosas, con capacidad de asombro, interesados en investigar y proponer. Una educación para ir más allá de la erudición o el enciclopedismo, enamorada de la labor de formar pensadores juiciosos, metódicos, siempre alertas en el mundo fáctico atiborrado de desafíos teóricos y prácticos. En suma, una educación para mentes ágiles, libres, perspicaces y productivas.

Empero, este aspecto era solo apenas una pequeña parte de las dimensiones de la Escuela Nueva, faltaba por tomarse en consideración su arista antropológica, aquella que indicaba que el proceso de enseñanza debía concentrarse en la única y particular naturaleza del infante. En otras palabras, la Escuela Nueva era una corriente pedagógica que adoptaba como principio la consideración de que el educando objeto de aprendizaje de la escuela y el colegio disfrutaba de una biología y psicología muy particular, incomparable con la "madurez" cognitiva y emocional del adulto, así como lo revela este punto de vista de Nieto Caballero:

Por fin el niño es el centro de todas las preocupaciones de la escuela. ¡Por fin el niño es el sol! Un nuevo concepto de la disciplina ha volcado las costumbres y mañas de la vieja escuela. La escuela nueva por sobre todo un amable desenvolvimiento del espíritu de cooperación social. Así, en las clases no se impone ya aquel trabajo aislado y silencioso de cada alumno, ni aquella torpe repetición en coro que nos vino de la escuela china. Reina allí la animación de un taller. Es aquella una verdadera colmena con su fecunda actividad, desordenada en apariencia. Hay poco orden, dice el mismo Dewey, cuando las cosas están en proceso de construcción (Nieto Caballero, 1937, p. 75).
Para terminar, indicando Anzola Gómez que la Escuela Nueva cumplía con el requisito de contar con una adecuada estrategia de desarrollo de las capacidades intelectuales porque aplicaba el método científico positivista en la enseñanza como se muestra a continuación:

La escuela que es una preparación para la vida, que debe antes que dar muchos conocimientos, preparar al niño para recibirlos, debe estar fundada en la vida misma, debe ser un reflejo de ella [...] una orientación hacia la observación de los hechos, un lugar inicial de experimentación que encamine hacia la sistematización, que prepare el espíritu para la captación elemental de los hechos con sus relaciones, causas, efectos [...] (Anzola-Gómez, 1930, p. 213).

Perspectiva que termina por corroborarse cuando estima que cada una de las ciencias que se convierten en las áreas del pensum deben impartirse en condiciones de apertura y con la preclara intención de enseñar a pensar, es decir, cultivar el intelecto, tal y como se observa a continuación, cuando toca el tema de cómo debería enseñarse geografía en la escuela: lejos de esto, el verdadero criterio que debe guiarnos, es hacer de ella una ciencia natural y educativa, esto es, procurar el desenvolvimiento de la inteligencia al par que se enseña la materia. Se trata, en pocas palabras, [...] de hacer comprender y hacer aprender (Anzola-Gómez, 1930, p. 187).

\section{CONCLUSIÓN}

Con el arribo del siglo XX sectores sociales provenientes de los estratos medio y alto de la sociedad colombiana aprovechan el clima de relativa tranquilidad política reinante después de 
la guerra de los mil días, el empeño empresarial por sentar las bases de un sólido proceso de industrialización, la presencia de inversión externa directa en el sector agrícola y minero y la expansión del gasto público hacia la implementación de una moderna y eficiente infraestructura vial y energética, para realizar el ejercicio juicioso de diagnosticar las causas por las cuales la educación básica y secundaria es de poca calidad y formular la estrategia que permita su rápida cualificación.

Este ejercicio teórico y práctico corrió por cuenta de personas como Agustín Nieto Caballero, Gabriel Anzola Gómez, Tomás Rueda Vargas, Miguel Jiménez, Simón Araujo, Tomás Cadavid, Rafael Bernal y Luis López de Mesa entre otros, quienes en múltiples y diversos escritos y ponencias expresaron que las causas del deplorable estado en el que se encontraba la educación de la infancia y adolescencia en Colombia era producto de una serie de factores convergentes.

Las explicaciones que estos intelectuales ofrecieron a la sociedad colombiana incluyeron, por ejemplo, argumentos de índole eugenésica como los presentados por Miguel Jiménez y Luis López de Mesa, de acuerdo con los cuales la educación no avanzaba por un asunto de degeneración racial. Aquí también cupieron argumentos de corte pragmático que consideraban la falta de reconocimiento social de la profesión docente y los bajos salarios del gremio como los elementos contundentes del fenómeno.

Así mismo, pudo notarse la presencia de puntos de vista que apuntaron a señalar la existencia de fallas estructurales en la formación de docentes en las normales y la considerable existencia de algunos sin ninguna clase de formación pedagógica o teórica, siendo más bien fruto de las circunstancias y de un acercamiento empírico al oficio, lo cual quedaba al descubierto con la gran cantidad de profesores sin título que ejercían la profesión en instituciones públicas y privadas de todos los departamentos del país.
Estos mismos intelectuales, vinculados con el paso del tiempo en distintos gobiernos, fueron partidarios de la organización de una institución de educación superior dedicada a la enseñanza de las ciencias de la educación, encargada de la formación metódica, teórica, conceptual, pedagógica y práctica de los profesores de los colegios de bachillerato y las escuelas de primaria, lo cual se vería materializado con la expedición del Decreto 1999 de 1933 que fundó la facultad de educación adscrita a la Universidad Nacional. Junto con lo cual llegó la apropiación y divulgación del discurso pedagógico de la Escuela Nueva como el enfoque educativo de la sociedad moderna, esa que esperaba que las nuevas generaciones vinieran dotadas de cualidad para resolver problemas con una invitación a la aplicación del método científico positivista y un marco librepensador lúdico.

\section{REFERENCIAS BIBLIOGRÁFICAS}

Álvarez, J. (2005). Luis López de Mesa y el debate en torno a la degeneración de la raza: cuerpo y educación en los albores del siglo XX. Revista Universidad de San Buenaventura. (23),271-285. Recuperado de: http://scienti. colciencias.gov.co:8084/publindex/docs/articulos/0121-7887/2291757/2306284.pdf

Anzola, G. (1930). Aspectos de la educación contemporánea. Bogotá, Colombia: Nueva Casa Editorial.

Bernal, R. (1949). La educación, he ahi el problema. Bogotá, Colombia: Ministerio de Educación Nacional

Cadavid, T. (1927). Informe que rinde el Director General de Instrucción Pública al senor Gobernador del Departamento con motivo de la reunión de la Asamblea en el año 1927. Medellín, Colombia: Imprenta Oficial.

Carvajal, M. (1926). Informe de la Inspección Seccional de Instrucción Pública de la segunda zona escolar. Revista de Instrucción Pública de Bolivar, (8), 86-98. 
Consejos prácticos a los maestros de escuela, (1918). Revista Departamental de Instrucción Pública de Antioquia, (9), 263-266.

Charry, C. (2011). Los intelectuales colombianos y el dilema de la construcción de la identidad nacional 1850-1930. Rewiew of Latin American and Caribbean Studies, (90), 55-70. Recuperado de: http://www.cedla.uva.nl/50. publications/pdf/revista/90revista europea/

Gómez, E. (1918). Extracto de los informes de Inspectores Provinciales. Revista Departamental de Instrucción Pública de Antioquia, (8), 247-248.

González, M. (1906). Informe de la visita practicada por el Inspector Provincial del suroeste. Revista Instrucción Pública Antioqueña, (1112), 362-367.

Helg, A. (2001). La educación en Colombia 1918-1957, una historia social, económica y política. Bogotá, Colombia: Universidad Pedagogica Nacional - Plaza y Janés.

Herrera, M. (1999). Modernización y Escuela Nueva en Colombia 1914-1951. Bogotá, Colombia: Universidad Pedagógica Nacional Plaza y Janes.

Holton, I. (1981). La Nueva Granada: veinte meses en los Andes. Bogotá: Banco de la República. Recuperado de: http://www.banrepcultural.org/blaavirtual/historia/viajeros/indice.htm

Informe de los inspectores provinciales. (1918) Revista Departamental de Instrucción Pública de Antioquia, (7), 219-220.

Jiménez, M. (1920a). Primera conferencia dictada en el Teatro Municipal de Bogotá el 21 de mayo de 1920 a la Asamblea de Estudiantes. En Los problemas de la raza en Colombia (pp.43-75). Bogotá, Colombia: Linotipos El Espectador.
Jiménez, M. (1920b). Algunos signos de degeneración colectiva en Colombia y en los países similares. En Los problemas de la raza en Colombia (pp.7-39). Bogotá, Colombia: Linotipos El Espectador.

Ministerio de Instrucción Pública. (1920). Memoria del Ministerio de Instrucción Pública al Congreso. (1920). Bogotá, Colombia: Imprenta La Luz.

Mora, A. (1918). Problemas escolares. Revista Departamental de Instrucción Pública de Antioquia, (4), 103-104.

Nieto, A (1915). Las Cajas Escolares y su acción en Bogotá. Bogotá, Colombia: Arboleda y Valencia.

Nieto, A. (1937). Sobre el problema de la educación nacional. Bogotá, Colombia: Editorial Minerva.

Ríos, R. (2004). Las ciencias de la educación en Colombia: algunos elementos históricos sobre su apropiación e institucionalización 19261954. Revista Memoria y Sociedad, (17), 73-86.

Rueda, T. (1945). El Gimnasio Moderno. Bogotá, Colombia: Editorial San Juan.

Tirado, A. (1985). Siglo y medio de bipartidismo. En M. Arrubla (Ed.), Colombia Hoy (pp. 102-145). Bogotá: Siglo XXI editores.

Torrejano, R. (2009). La agitación social y laboral en Colombia 1902-1934. Bogotá, Colombia: Corporación Universitaria Republicana.

Vega, R. (2002). Gente muy rebelde. V. 1. Bogotá, Colombia: Ediciones Pensamiento Crítico.

Villegas, J. (1914). Conferencia del Inspector General. Revista de Instrucción Pública del Valle del Cauca, (20), 236-239.

Villegas, J. (1915). Informe de la provincia de Buga. Revista de Instrucción Pública del Valle del Cauca, (35), 499-500. 